\title{
Topological Hysteresis in the Intermediate State of Type-I Superconductors
}

\author{
Ruslan Prozorov, ${ }^{1}$ Russell W. Giannetta, ${ }^{2}$ Anatolii A. Polyanskii, ${ }^{3}$ and Garry K. Perkins ${ }^{4}$ \\ ${ }^{1}$ Ames Laboratory and Department of Physics 8 Astronomy, \\ Iowa State University, Ames, Iowa 50011, U.S.A. \\ ${ }^{2}$ Loomis Laboratory of Physics, University of Illinois at Urbana - Champaign, \\ 1110 West Green Street, Urbana, IL 61801, U.S.A. \\ ${ }^{3}$ Applied Superconductivity Center, University of Wisconsin, \\ 1500 Engineering Drive, Madison, WI 53706, U.S.A. \\ ${ }^{4}$ Blackett Laboratory, Imperial College of Science Technology and Medicine, London SW7 2BZ, United Kingdom
}

(Dated: 7 July 2005)

\begin{abstract}
Magneto-optical imaging of thick stress-free lead samples reveals two distinct topologies of the intermediate state. Flux tubes are formed upon magnetic field penetration (closed topology) and laminar patterns appear upon flux exit (open topology). Two-dimensional distributions of shielding currents were obtained by applying an efficient inversion scheme. Quantitative analysis of the magnetic induction distribution and correlation with magnetization measurements indicate that observed topological differences between the two phases are responsible for experimentally observable magnetic hysteresis.

PACS numbers: 74.25.Ha,74.25.Op,89.75.Kd
\end{abstract}

The structure of the intermediate state in type-I superconductors has a long history beginning with the pioneering papers of Landau 1, 2 2 and continuing to the present day 3, 4, 5, 6, 7, 8, 9, 10, 11, 12]. Intermediate state flux patterns closely resemble those found in a wide variety of hydrodynamic, chemical and solid state systems [9, 13, 14]. Study of the intermediate state is therefore vital to a general understanding of pattern formation. Flux structures can be readily tuned with a magnetic field and imaged with magneto-optical (MO) techniques [3, 4]. The observed patterns can then be correlated with underlying thermal, magnetic and resistive properties. Early MO images of the intermediate state revealed a variety of phenomena not predicted by the simple theory 3, 44. The initial models were then refined to include domain branching and corrugation. Still, it is widely believed that a true thermodynamically stable configuration of the intermediate state is the famous Landau laminar structure [2].

Magnetic hysteresis is routinely observed in type-I superconductors and has generally been attributed to impurities, grain boundaries, dislocations and other imperfections of the crystal structure [4]. In this Letter we focus on the relationship between the topology of flux structures and their macroscopic magnetic properties. We find that a small residual hysteresis remains even in the most carefully prepared samples and present evidence that this hysteresis arises from the differences in the topology of the intermediate state between flux entry and flux exit.

Samples were prepared from 99.9999\% lead 23] foils and rods. More than a dozen samples were prepared by using various annealing protocols or deliberately introducing stress by cold rolling. The most reversible samples were obtained by melting lead between two Pyrex slides.
Samples had thickness between $d=0.1$ and $1.5 \mathrm{~mm}$ and were about $1.5 \times 1.5 \mathrm{~mm}^{2}$ in planar dimension. The topological features described here were thickness independent above $d \approx 0.5 \mathrm{~mm}$, which indicates that they are not due to surface-related effects. We show data for samples which had demagnetization factors of about $N=0.5$ (determined both from initial magnetisation and direct calculations [20].) Quantum Design MPMS magnetometer was used for DC magnetization measurements. MO imaging was performed in a pumped flow-type optical ${ }^{4} \mathrm{He}$ cryostat using Faraday rotation of polarized light in Bi-doped iron-garnet films with in-plane magnetization [15]. In all images the bright regions correspond to the normal state and dark regions to the superconducting state.

Figure 1shows typical magnetization loops for the cold - rolled sample (top panel) and the most reversible sample of similar dimensions (bottom). The stressed sample shows considerably more hysteresis than the stress-free sample. The hysteresis increases for decreasing field in the stressed sample as expected from pinning, whereas the hysteresis disappears approaching $H=0$ in the reversible sample, indicating complete Meissner expulsion. In that sample pinning is also absent at larger fields as well. Also shown in Fig 1 are MO images taken at the same temperature and magnetic fields indicated by arrows. The flux structure for the stressed sample is dendritic and a significant amount of flux is trapped at $H=0$. In the stress-free sample the patterns are noticeably different, revealing flux tube phase upon flux penetration and well-defined laminar pattern upon flux exit. This behavior was observed at all accessible temperatures.

Figure 2 shows details of the evolution of flux patterns in the stress-free sample at $T=6 \mathrm{~K}$. After cooling in zero 


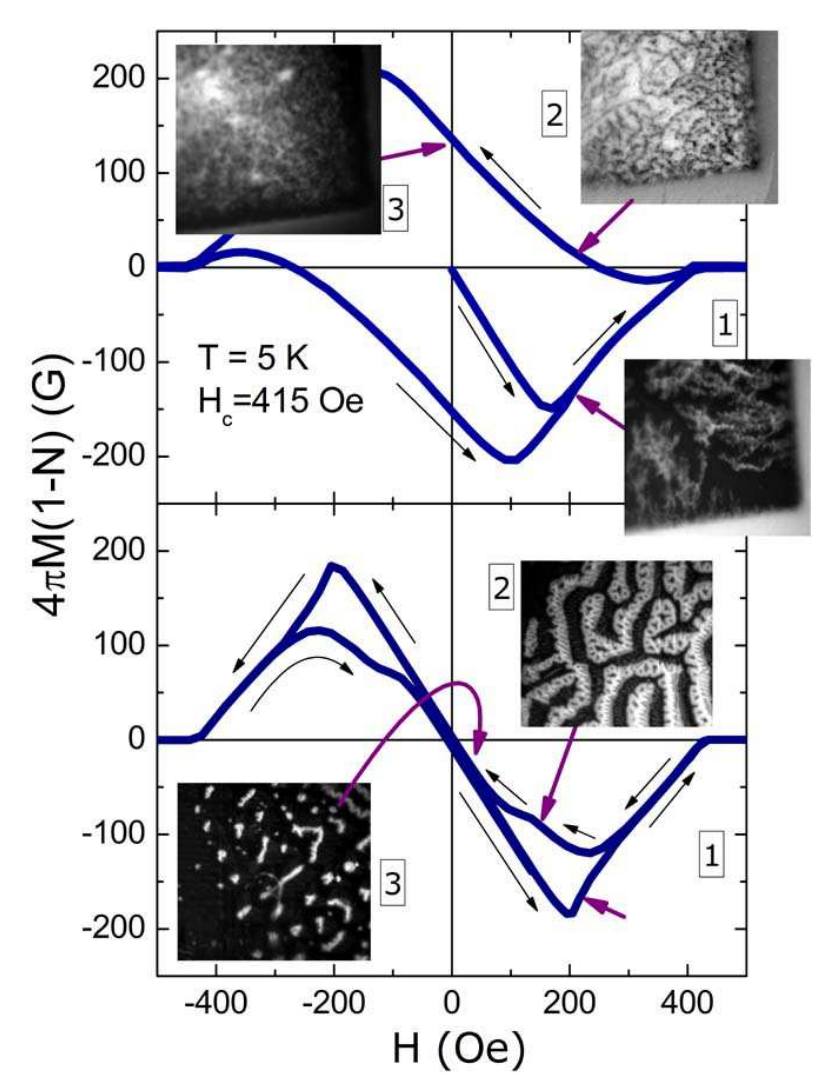

FIG. 1: Magnetization loops in stressed (top) and stress-free sample (bottom). Also shown MO images obtained at magnetic fields indicated by arrows ( the numbers indicate images in succession).

field, a full magnetization loop was measured with a maximum magnetic field exceeding $H_{c} \approx 260$ Oe. Shown by the solid line, the $M(H)$ loop exhibits magnetic hysteresis at intermediate fields. The crucial question is whether this hysteresis is due to extrinsic factors (defects or residual stress) or it is an intrinsic property of the intermediate state. To clarify this, we performed zero-field and field - cooling experiments. The results are shown by the symbols in Fig. 2] The circles are obtained after cooling in zero field to $6 \mathrm{~K}$ and then increasing field, whereas squares indicate measurements after cooling the sample to $6 \mathrm{~K}$ in a particular field. If the hysteresis were due to pinning, the zero field cooled circles should coincide with the ascending branch of the $M(H)$ loop but field-cooled squares should not. Instead, Fig 2 clearly shows that both circles and squares coincide exactly with the directly measured magnetization loop, implying that the hysteresis is not due to pinning. The fact that the ascending and descending branches merge at small and large fields is also inconsistent with pinning. Also, we observed no magnetic relaxation with either flux penetration or flux exit. These results strongly suggest that the hysteresis in stress-free samples is due to the topological difference between the closed flux tube phase and the open laminar

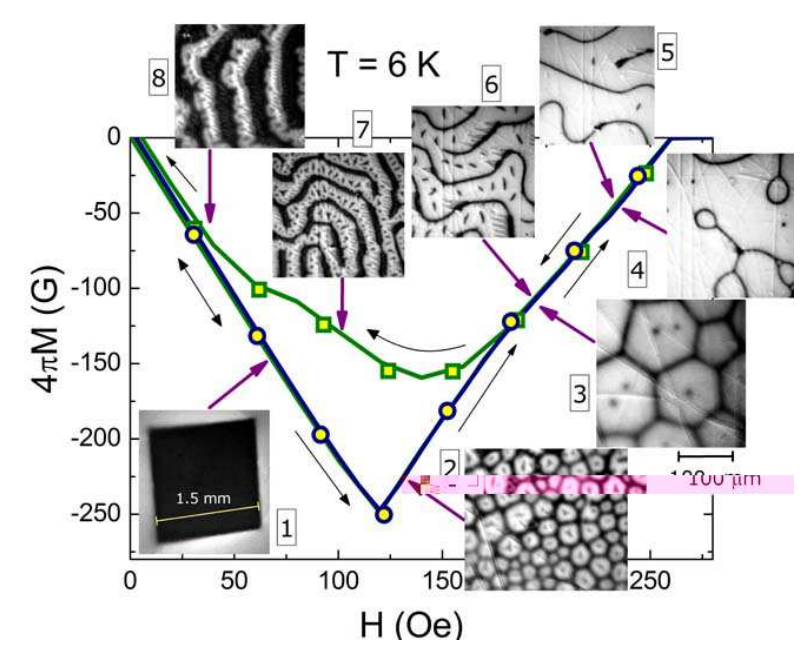

FIG. 2: Magnetization loop in a stress-free sample accompanied by MO and zfc-fc measurements (see text). Circles are obtained after applying field after cooling in zero field. Squares show results of field-cooled measurements.

phase.

MO images shown in Fig. 2] reveal that after pure Meissner screening, the intermediate state appears, not as laminae, but as an assembly of normal tubes carrying magnetic flux and separated by superconducting regions. (MO image $\sharp 1$ shows the entire sample, others zoom in to reveal the structure). These images show that flux tubes have a variety of structures - from simple monodomain to complex objects threaded with superconducting tubes. The flux tube phase favors hexagonal symmetry, almost exactly as modeled by Goren and Tinkham [16]. In all cases, this tubular phase has a closed topology that allows screening currents to circulate [4]. Similar patterns were directly observed in In [3, 8], Re [3], Sn [3, 22] and $\mathrm{Hg}$ [4] and it seems that closed topology tubular pattern is a generic feature of the intermediate state of pinningfree type-I superconductors upon flux penetration.

Another possibility for the hysteresis is the edge barrier for flux penetration (including both, Bean-Livingston and geometric barriers) 17, 18, 19. However, such a barrier would result in delayed flux penetration and more negative values of magnetization compared to the thermodynamic values. Figure 2 shows that in our samples penetration occurs at the thermodynamic field $H_{c}(1-N) \approx 120$ Oe and magnetization at that point is as supposed to be in thermodynamic equilibrium, $4 \pi M=-H_{c}$, independent of the demagnetization factor. We attribute the weak influence of the edge barrier to the large thickness of our samples. On the other hand, the edge barrier could be involved in the formation of the observed topologies. When small fingers of the normal phase are formed at the sample edge, the surface barrier will prevent their continuous penetration into the interior and will break them into small flux tubes as suggested in 


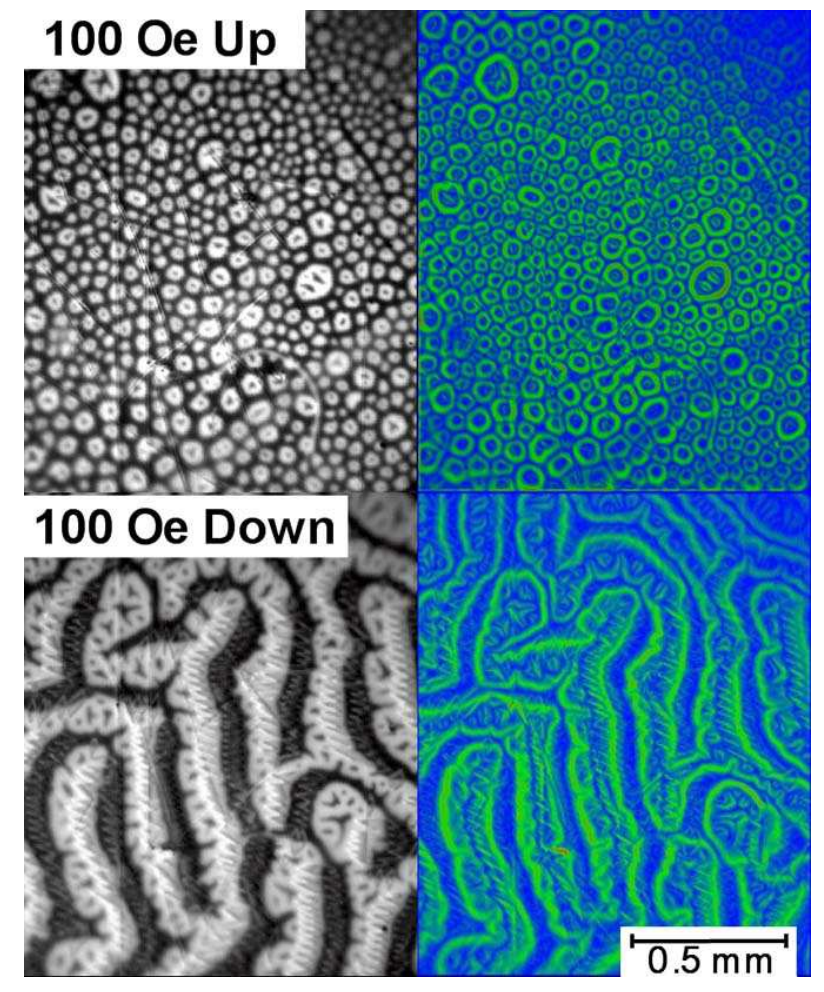

FIG. 3: $T=5 \mathrm{~K}, H=100$ Oe: (left) Distribution of the magnetic induction upon flux penetration (top) and exit (bottom);(right) corresponding patterns of shielding currents density obtained by numerical inversion. Intensity is proportional to the current density. (Color online)

\section{Ref. 17].}

Furthermore, quantitative imaging of the magnetic induction can be used to visualize spatial distribution of shielding currents. This experimental information is important for theoretical analysis involving current-loop models [10, 11] as well as for general understanding of pattern formation in type-I superconductors. We used recently developed fast inversion scheme 21]. Figure 3 shows the result obtained for $H=100$ Oe. Left images correspond to flux penetration (top) and flux exit (bottom) in our most reversible sample. The right panel shows corresponding distributions of the shielding currents density (proportional to the brightness). Clearly, current distribution exhibits two topologically distinct patterns. The closed topology of small current loops vs. open topology of branched current streams.

The direction of the currents flow is seen in Fig 4 which shows the reconstruction at $H=300$ Oe. At this field the features are larger and contour lines with directional arrows can be used to better visualize the flow patterns. In both topologies, currents flow counterclockwise - against the direction of Meissner currents flowing along the sample edges. At low fields Meissner currents dominate and hysteresis is negligible. It appears only at intermediate fields when the intermediate state consists

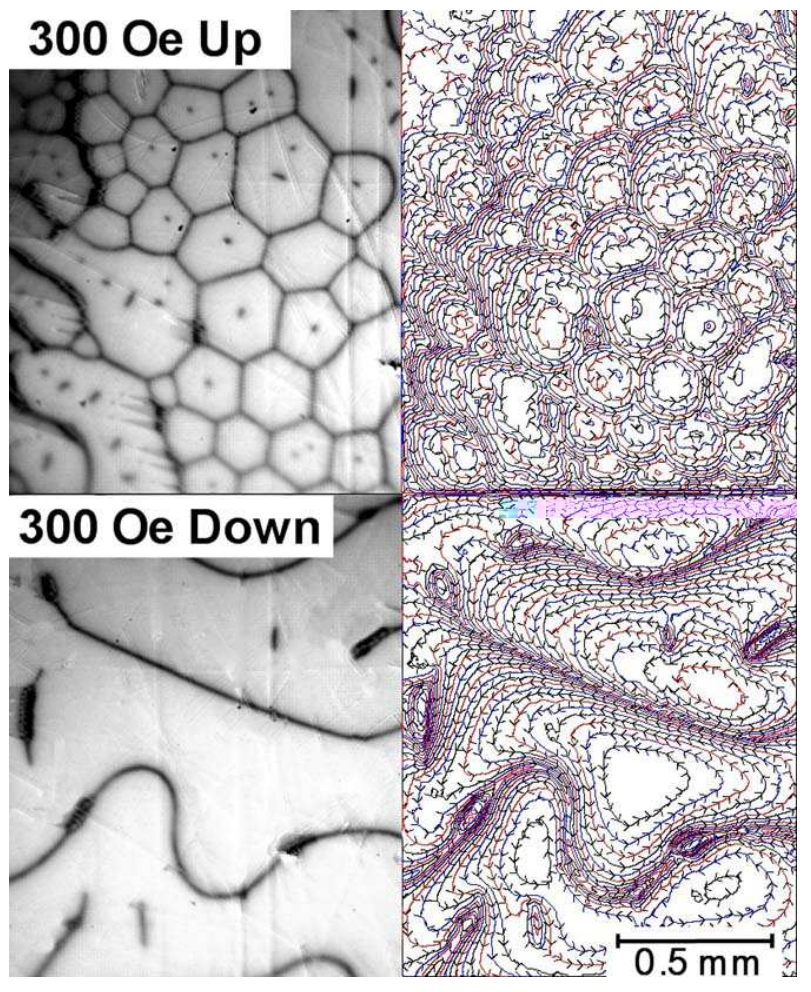

FIG. 4: $T=5 \mathrm{~K}, H=300$ Oe: (left) Distribution of the magnetic induction upon flux penetration (top) and exit (bottom);(right) corresponding contour plots of shielding currents density obtained by the numerical inversion. Arrows show the direction of currents. (Color online)

of mobile ensemble of flux tubes on field entry and laminar structure that forms escape paths for flux expelled by the Meissner effect upon flux exit.

To evaluate quantitative correspondence of the $\mathrm{MO}$ and $M(H)$ measurements we calculate total magnetic moment from the $\mathrm{MO}$ images by using $4 \pi M=$ $\int[\mathbf{H}-\mathbf{B}(\mathbf{r})] d^{3} \mathbf{r}$. Magnetic induction is linearly proportional to the intensity, hence integrating images and using initial slope of the measured $M(H)$ loop for calibration, $M$ is obtained. Figure 5 shows that $M(H)$ loop from the MO images (solid symbols) is in a good agreement with the direct measurements (open symbols).

Observed topological hysteresis is also clearly seen on the profiles of the magnetic induction. Following Landau 2], it has generally been assumed that the magnetic field inside the normal phase in the intermediate state is close to $H_{c}[3,4,9,17]$. Figure 5 shows profiles of the magnetic induction measured at the same external field in the flux tube phase (obtained on flux entry) and in a laminar phase (obtained on flux exit). While the field in the laminae is comparable to the critical field $H_{c}$ as expected, the field above the flux tubes is much smaller. Indeed, the measurements are carried out $\approx 10 \mu \mathrm{m}$ above the sample, so the measured field is reduced compared to the values inside the tubes. However, simple numerical 


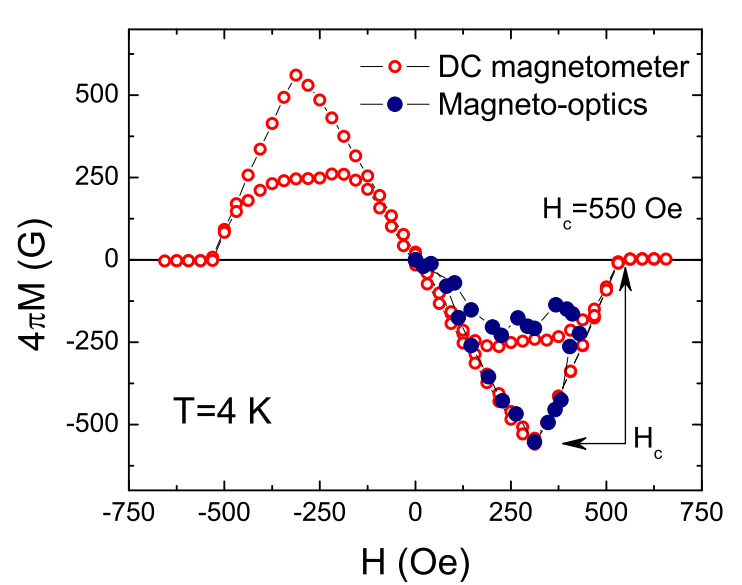

FIG. 5: Comparison of DC magnetization measured by magnetometer and reconstructed from MO images. Arrows show that at the minimum $4 \pi M=-H_{c}$ as expected at thermodynamic equilibrium without edge barriers.

analysis with appropriate dimensions does not reproduce such substantial reduction. It is possible that flux tubes widen when approaching the surface [3], but why it is not seen in the laminar phase?

Alternatively, it is possible that when a flux tube initially appears at the sample edge with critical magnetic field inside. Due to closed topology, the total magnetic flux in such tube is now conserved. The nucleated flux tube is driven by Meissner currents toward sample interior (these currents flow everywhere on the surfaces perpendicular to the magnetic field [20]) until it is stopped at the center or later by other tubes piling up from the center outward. When the flux tube reaches the interior, its radius may increase to minimize the magnetic field energy. Real time imaging showed that flux tubes produced at the sample edge continue to travel toward the center and form an apparently outwardly expanding phase 22], as seen in the lower-right panel of Fig [6] The real-time observations were first made by Solomon and Harris in 1971 22. The rigorous evaluation of the free energy of even a single tube is not simple. The difficulty is the lack of a sharp interface between the superconductor and tube interior, which decreases the surface energy. The tubes repel each other at the large distances due to interaction of screening currents, but they attract each other when their "cores" overlap. When tubes merge, average magnetic field in the tube increases until it reaches $H_{c}$. At this stage, the honeycomb lattice is formed and it persists almost up to $H=H_{c}$. The observed topological hysteresis is observed only at the stage when magnetic field inside the tubes is less than $H_{c}$ and before the formation of rigid hexagonal lattice, Fig 2. A detailed study of tube nucleation and expansion is needed to quantify this issue.

We thank J. R. Clem, A. T. Dorsey, T. A. Girard,

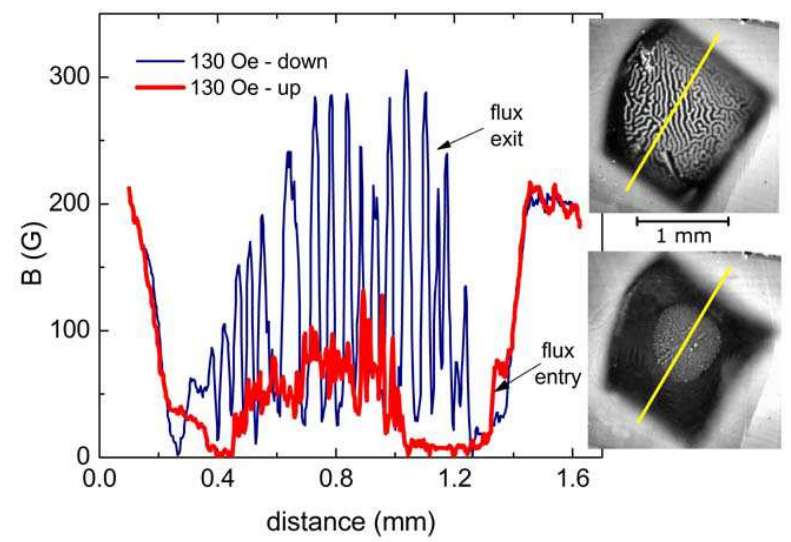

FIG. 6: Profiles of the magnetic induction measured at $T=4$ $\mathrm{K}$ in the flux tube phase (flux penetration)and laminar phase (flux exit). Right panel shows corresponding MO images.

N. D. Goldenfeld, R. P. Huebener, R. V. Kohn, D. C. Larbalestier and V. K. Vlasko-Vlasov for useful discussions.

[1] L. D. Landau, Sov. Phys. JETP 7, 371 (1937).

[2] L. Landau, Nature 141, 688 (1938).

[3] J. D. Livingston and W. DeSorbo, in Superconductivity, edited by R. D. Parks (Marcel Dekker, Inc., New York, 1969), Vol. 2, p. 1235.

[4] R. P. Huebener, "Magnetic Flux Structures of Superconductors" (Springer-Verlag, New-York, 2001).

[5] F. Liu, M. Mondello, and N. Goldenfeld, Phys. Rev. Lett. 66, 3071 (1991).

[6] H. Frahm, S. Ullah, and A. T. Dorsey, Phys. Rev. Lett. 66, 3067 (1991).

[7] C. R. Reisin and S. G. Lipson, Phys. Rev. B 61, 4251 (2000).

[8] V. Jeudy, C. Gourdon, and T. Okada, Phys. Rev. Lett. 92, 147001 (2004).

[9] R. Choksi, R. V. Kohn, and F. Otto, J. Nonlinear Science 14, 119 (2004).

[10] R. E. Goldstein, D. P. Jackson, and A. T. Dorsey, Phys. Rev. Lett. 76, 3818 (1996).

[11] A. T. Dorsey and R. E. Goldstein, Phys. Rev. B 57, 3058 (1998).

[12] O. Narayan, Phys. Rev. Lett. 81, 5035 (1998).

[13] P. Coullet and P. Huerre, "New Trends in Nonlinear Dynamics and Pattern Forming Phenomena", (Plenum Press, New York, 1991).

[14] D. Walgraef, "Spatio-Temporal Pattern Formation", (Springer, New York, 1997).

[15] L. A. Dorosinskii et al., Physica C 203, 149 (1992).

[16] R. N. Goren and M. Tinkham, J. Low. Temp. Phys. 5, 465 (1971).

[17] A. Fortini and E. Paumier, Phys. Rev. B 14, 55 (1976).

[18] J. R. Clem, R. P. Huebener, and D. E. Gallus, J. Low Temp. Phys. 12, 449 (1973).

[19] H. Castro et al., Phys. Rev. B 59, 596 (1999). 
[20] R. Prozorov et al., Phys. Rev. B 62, 115 (2000).

[21] G. K. Perkins, Yu. V. Bugoslavsky and A. D. Caplin, Supercond. Sci. Technol. 15, 1140 (2002).

[22] P. R. Solomon and R. E. Harris, (United Aircraft Re- search Laboratories, (videotape provided by Dr. N. D. Goldenfeld), 1971).

[23] various sources, including Puratronic ${ }^{\circledR}$ from Alfa Aesar 